\title{
Dual specificity phosphatase 6 as a new therapeutic target candidate for epithelial ovarian cancer
}

\author{
Seiya Sato ${ }^{1}$, Hiroaki Itamochi ${ }^{2}$ \\ ${ }^{1}$ Department of Obstetrics and Gynecology, Shimane University School of Medicine, Izumo, Japan; ${ }^{2}$ Department of Clinical Oncology, Iwate \\ Medical University School of Medicine, Yahaba, Japan \\ Correspondence to: Hiroaki Itamochi, MD, PhD. Department of Clinical oncology, Iwate Medical University School of Medicine, 1-1-1 Idaidori, \\ Yahaba-cho, Shiwa District, Iwate 028-3694, Japan. Email: itamochi@hotmail.com. \\ Provenance: This is an invited article commissioned by the Executive Editor Dr. Zhi-De Hu (Department of Laboratory Medicine, the Affiliated \\ Hospital of Inner Mongolia Medical University, Hohhot, China). \\ Comment on: James NE, Beffa L, Oliver MT, et al. Inhibition of DUSP6 sensitizes ovarian cancer cells to chemotherapeutic agents via regulation of \\ ERK signaling response genes. Oncotarget 2019;10:3315-27.
}

Submitted Oct 27, 2019. Accepted for publication Dec 16, 2019.

doi: 10.21037/atm.2019.12.105

View this article at: http://dx.doi.org/10.21037/atm.2019.12.105

\section{Introduction}

Epithelial ovarian cancer (EOC) is a fatal disease for which methods of early detection are not yet available (1). The current standard treatment for EOC is tumor debulking surgery and systemic chemotherapy consisting of taxane and platinum-based agents (2). In recent years, new therapeutic agents against advanced EOC have emerged in the clinical setting such as bevacizumab, which is a humanized monoclonal antibody targeting vascular endothelial growth factor-A, and poly (ADP-ribose) polymerase inhibitors for patients with $B R C A$ mutations (3-5). Nevertheless, there has been no significant improvement in the overall survival of patients with advanced EOC over the last decade. EOC acquire resistance to chemotherapy consisting of DNAdamaging agents, which leads to recurrence and makes it difficult for patients to achieve a cure (6). Various clinical and pre-clinical strategies such as immune checkpoint blockade, targeted therapy against cancer stem cells and cell cycle checkpoint kinase are being studied to overcome drug resistance (7). However, no breakthrough in EOC treatment has been reported and new treatment strategies are needed.

\section{Dual specificity phosphatase 6 (DUSP6) and mitogen-activated protein kinases (MAPKs)}

Understanding tumor molecular biology is essential for selecting the best strategies for targeted therapy (8). Among them, molecular therapy targeting the MAPK signaling pathway has been well studied. MAPKs, which include extracellular signal-regulated protein kinases (ERKs), play an important role in physiological processes such as proliferation, differentiation, apoptosis and stress responses, not only in normal cells but also in cancer cells. Aberrant MAPK pathway signaling is involved in carcinogenesis and progression in many cancers $(9,10)$. DUSP6 is a member of the MAPK phosphatase family that deactivates ERKs via negative feedback (Figure 1). Over the last few decades, evidence has emerged that DUSP6 is involved in many normal cellular functions (11). In addition, numerous studies have shown that DUSP6 is associated with carcinogenesis and progression in cancers (12). DUSP6 is also dynamically involved in DNA damage repair pathways and stress responses; this involvement indicates that it plays an important role in cancer cell chemoresistance (13).

\section{DUSP6 and cancer}

DUSP6 plays a role as a tumor suppressor through dephosphorylation of p38, JNK and ERK (14), which are the major molecules in the MAPK signaling pathway. Activation of ERKs in cancer cells induces DUSP6 transcription, and DUSP6 suppresses MAPK signaling by negative feedback (15). Interestingly, DUSP6 also functions 
Page 2 of 5

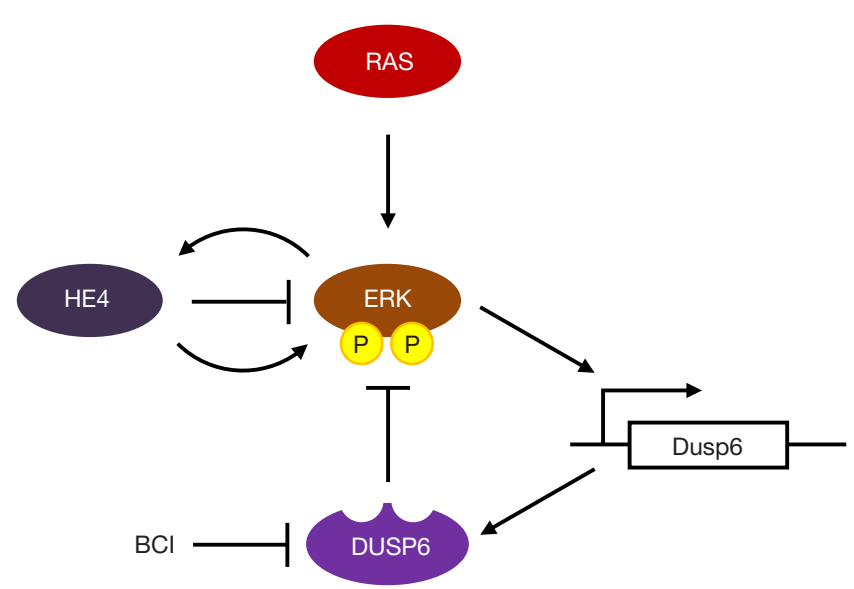

Figure 1 Interaction between DUSP6 and ERK signaling pathways. HE4, human epididymis protein 4; ERK, extracellular signal-regulated protein kinase; DUSP6, dual specificity phosphatase 6; BCI, (E)-2-benzylidene-3-(cyclohexylamino)-2,3dihydro-1H-inden-1-one.

as an oncogene by helping tumor cells adapt to abnormally enhanced MAPK signaling. Since DUSP6 has two roles as described above, there is a difference in the expression of DUSP6 depending on the type of cancer. For example, decreased expression of DUSP6 has been observed in pancreatic cancer and non-small cell lung cancer, and it has been suggested that DUSP6 acts as a tumor suppressor in these cancers (12). In contrast, it has been reported that DUSP6 is upregulated in gastric cancer, breast cancer and acute myeloid leukemia (12). It has been hypothesized that DUSP helps these malignancies to adapt to the excessive proliferative growth signals, and inhibition of DUSP6 has been reported to show antitumor effects. DUSP inhibition is thought to rapidly overactivated MAPK signaling, causing activation of effectors such as p53, ATM, and $\mathrm{CHK} 1 / 2$, reduce cell proliferation, and induce cell death. Therefore, DUSP6 is attracting attention as a therapeutic target because its inhibition may have an antitumor effect in neoplasms with enhanced MAPK. For example, novel preclinical data showed the antitumor effect of DUSP6 inhibition in highly aggressive malignant peripheral nerve sheath tumors (MPNSTs) in which MAPK signaling plays an important role in tumorigenesis (16). When DUSP6 was knocked down using small hairpin RNA, overexpression of ERK and c-Jun N-terminal kinases (JNK) was induced and proliferation of MPNST cells was suppressed. Furthermore, exposure to (E)-2-benzylidene-3-(cyclohexylamino)2,3-dihydro-1H-inden-1-one (BCI), a specific allosteric
Sato and Itamochi. DUSP6 as a therapeutic target for ovarian cancer

inhibitor of DUSP6, decreased MPNST cell line survival through JNK activation. In an in vivo model using MPNST cells, BCI treatment also increased tumor ERK and JNK activation and showed antitumor effects (16).

\section{DUSP6 and ovarian cancer}

So far, the relationship between EOC and DUSP6 has not been fully elucidated. Preclinical data on the potential of DUSP6 as a therapeutic target for ovarian cancer was first reported in 2008 (17). In that study, the authors showed that the expression of DUSP6, a negative regulator of the ERK pathway, was decreased in ovarian cancer cells with enhanced ERK pathway. The cause was ubiquitination/ proteasome degradation mediated by accumulation of intracellular reactive oxygen species such as hydrogen peroxide. In addition, when DUSP6 in ovarian cancer cells was knocked down using small hairpin RNA, cell proliferation ability via ERK pathway was upregulated and resistance to cisplatin was induced. Furthermore, induction of DUSP6 in ovarian cancer cells sensitized cisplatininduced apoptosis both in vitro and in vivo. These results suggest that an abnormal increase in reactive oxygen species in ovarian cancer cells decreases the function of DUSP6 protein, thereby activating the ERK pathway and inducing drug resistance in ovarian cancer cells.

Recently, James et al. (18) reported interesting basic experimental data that raised the possibility of DUSP6 as a new therapeutic target for EOC. The authors reported that inhibition of DUSP6 increased the sensitivity of ovarian cancer cells to paclitaxel and carboplatin through regulation of ERK signaling. They found that the mechanism of this combined effect was upregulation of EGR1 gene expression, which is a promoter of apoptosis, and downregulation of c-JUN gene expression, which is one of the proto-oncogenes. These phenomena were opposed by recombinant human epididymis protein 4 (HE4), which suggests that a relationship exists between DUSP6 and HE4 in ovarian cancer cells in vitro. HE4 is a secreted glycoprotein with a molecular weight of approximately $25 \mathrm{kDa}$, which was found in epithelial cells distal to the epididymis. Serum HE4 levels in ovarian malignant tumors are significantly higher than levels in ovarian benign tumors, which points to its potential use as a biomarker of ovarian cancer. These findings showed that DUSP6 and HE4 are both associated with the ERK pathway. In a separate study, the authors reported that HE4, which is highly expressed in ovarian cancer cells, is involved in immune 
evasion of ovarian cancer cells by attenuating immune cell function through upregulation of DUSP6 (19). Thus, they attempted to elucidate the relationship between DUSP6 and HE4 by conducting in vitro experiments using ovarian cancer cell lines (18). Interestingly, immunohistochemistry revealed that the expression intensity of DUSP6 in serous ovarian cancer tissues was higher than that in normal ovarian tissues, and that $\mathrm{HE} 4$ protein expression was increased and showed a positive correlation with DUSP6 protein expression. From this result, they concluded that inhibition of DUSP6 is a promising therapeutic strategy in serous ovarian cancer associated with HE4. Further research on the relationship between HE4 and DUSP6 in carcinogenesis and drug resistance in ovarian cancer is expected to clarify the therapeutic potential of DUSP6. Another interesting finding in the article by James et al. (18) is that the gene profile induced in cancer cells differs depending on exposure to BCI and chemotherapy drug. Using qPCR array of Human Cancer Drug Resistance (Qiagen), they compared the gene expression profiles of ovarian cancer cell lines in different groups: exposure to BCI alone, BCI plus carboplatin, or BCI plus paclitaxel. Analysis using a heat map showed similarities in gene expression signatures when cells were exposed to either BCI alone or carboplatin alone, but the expression signature was clearly unique when cells were treated with paclitaxel alone. Of note, the ERBB3 gene was downregulated in the groups treated with the combination of BCI plus paclitaxel or carboplatin. The significance of this result in drug resistance of ovarian cancer is unclear. However, it is valuable data showing that the stress response in the tumor differs depending on exposure to chemotherapeutic drug. It is currently difficult to accurately identify the response genes induced in tumors when patients with EOC are treated with standard chemotherapy consisting of paclitaxel and carboplatin. In addition, it is unclear whether the cellular response in vitro actually occurs in vivo when the drug is administered at the dose calculated in the clinical setting. More efficient targeted therapy may be achieved if it is possible to regularly monitor changes in gene expression in tumors that respond to multiple anticancer drugs using analysis of circulating tumor cells or cell-free DNA.

\section{Is DUSP6 a target for ovarian cancer treatment?}

As noted above, there is limited preclinical data on carcinogenicity and drug resistance in ovarian cancer. Therefore, it is currently unclear whether DUSP6 is actually a good target for ovarian cancer treatment. How can treatment of EOC targeting DUSP6 be clinically applied in the future? As mentioned in the beginning, DUSP6 plays a pivotal tumor suppressive role through negative feedback of MAPK signaling in the carcinogenesis of ovarian cancer. In other words, inhibition of DUSP6 may contribute to the development of ovarian cancer. In fact, it has been suggested that nuclear DUSP6 in isolated circulating tumor cells is involved in brain metastasis of triple negative breast cancer patients (20). Therefore, detailed functional analysis of DUSP6 in ovarian cancer is required in order to apply DUSP6 inhibition to the treatment of ovarian cancer. Specifically, it is necessary to attenuate the ERK-induced signals in cancer by administration of DUSP6 inhibitors (21). Furthermore, it is necessary to confirm whether administration of DUSP6 inhibitor together with a cytotoxic drug to patients actually inhibits the growth of ovarian cancer cells in vitro and in vivo. To our knowledge, there are no DUSP6 inhibitors that can be used safely in the clinical setting. DUSP inhibition may activate signal transduction pathways that can cause serious side effects and secondary carcinogenesis. If other molecules functionally similar to DUSP6 that negatively regulate ERKs are discovered, they may be used in cancer treatment strategies. Conversely, if DUSP6 can be introduced by gene therapy into the tumor only, it may be effective against ovarian cancer whose growth depends on MAPK signaling.

In the current era of precision medicine, the development of cancer treatments relies on cancer genetic information. Cancer genetic abnormalities have been comprehensively revealed with the help of innovative technologies such as next-generation sequencers. Thanks to clinical sequencing, the heterogeneity of cancer has been revealed. In addition, these data enable us to obtain a large amount of genomewide profiling information from cancer and give rise to the possibility of personalized cancer therapy. However, systematic evaluation of these results has not led to the generation of new targeted therapies. Applying in vitro data to clinical practice has long been a challenge. Historically, oncogene amplification has been reported in many cancers (22) including ovarian cancer, and it has been used to develop targeted therapies under the concept of oncogene addiction (23). If the overexpressed driver gene is suppressed in cancer cells by targeted therapy, it will lead to cell death via apoptosis (24). This strategy has been effective in a limited number of cancer patients and this is thought to be due to cancer heterogeneity and the existence of 
various drug resistance mechanisms. Furthermore, because the driver genes in cancer interact, negative or positive feedback might be triggered by the interaction with another pathway despite suppression of one pathway. Therefore, recent cancer treatments have been developed based on the concept of synthetic lethality (25). For the above reasons, it is desirable to develop drugs that achieve synthetic lethality with DUSP6 inhibition.

\section{Conclusions}

It is uncertain whether ovarian cancer treatment targeting DUSP6 can be clinically applied in the future. To investigate this further, it will be necessary to perform detailed functional analysis of DUSP6 in ovarian cancer, develop inhibitors that can be administered to humans, and search for drugs that show synthetic lethality in combination with DUSP inhibitors. We hope that treatment strategies targeting DUSP6 will lead to improved prognosis in patients with advanced EOC.

\section{Acknowledgments}

None.

\section{Footnote}

Conflicts of Interest: The authors have no conflicts of interest to declare.

Ethical Statement: The authors are accountable for all aspects of the work in ensuring that questions related to the accuracy or integrity of any part of the work are appropriately investigated and resolved.

\section{References}

1. Siegel RL, Miller KD, Jemal A. Cancer statistics, 2019. CA Cancer J Clin 2019;69:7-34.

2. Sato $\mathrm{S}$, Itamochi H. Neoadjuvant chemotherapy in advanced ovarian cancer: latest results and place in therapy. Ther Adv Med Oncol 2014;6:293-304.

3. Fong PC, Boss DS, Yap TA, et al. Inhibition of poly(ADPribose) polymerase in tumors from BRCA mutation carriers. N Engl J Med 2009;361:123-34.

4. Burger RA, Brady MF, Bookman MA, et al. Incorporation of bevacizumab in the primary treatment of ovarian cancer. N Engl J Med 2011;365:2473-83.
5. Perren TJ, Swart AM, Pfisterer J, et al. A phase 3 trial of bevacizumab in ovarian cancer. $\mathrm{N}$ Engl $\mathrm{J}$ Med 2011;365:2484-96.

6. Sato S, Itamochi H. Ovarian Cancer and Drug Resistance. Curr Obstet Gynecol Rep 2015;4:18-25.

7. Zamarin D. Novel therapeutics: response and resistance in ovarian cancer. Int J Gynecol Cancer 2019;29:s16-21.

8. Itamochi H. Targeted therapies in epithelial ovarian cancer: Molecular mechanisms of action. World J Biol Chem 2010;1:209-20.

9. Rezatabar S, Karimian A, Rameshknia V, et al. RAS/ MAPK signaling functions in oxidative stress, DNA damage response and cancer progression. J Cell Physiol 2019. [Epub ahead of print].

10. Papa S, Choy PM, Bubici C. The ERK and JNK pathways in the regulation of metabolic reprogramming. Oncogene 2019;38:2223-40.

11. Kidger AM, Keyse SM. The regulation of oncogenic Ras/ERK signalling by dual-specificity mitogen activated protein kinase phosphatases (MKPs). Semin Cell Dev Biol 2016;50:125-32.

12. Ahmad MK, Abdollah NA, Shafie NH, et al. Dualspecificity phosphatase 6 (DUSP6): a review of its molecular characteristics and clinical relevance in cancer. Cancer Biol Med 2018;15:14-28.

13. Bagnyukova TV, Restifo D, Beeharry N, et al. DUSP6 regulates drug sensitivity by modulating DNA damage response. Br J Cancer 2013;109:1063-71.

14. Caunt CJ, Keyse SM. Dual-specificity MAP kinase phosphatases (MKPs): shaping the outcome of MAP kinase signalling. FEBS J 2013;280:489-504.

15. Pratilas CA, Taylor BS, Ye Q, et al. (V600E)BRAF is associated with disabled feedback inhibition of RAFMEK signaling and elevated transcriptional output of the pathway. Proc Natl Acad Sci U S A 2009;106:4519-24.

16. Ramkissoon A, Chaney KE, Milewski D, et al. Targeted Inhibition of the Dual Specificity Phosphatases DUSP1 and DUSP6 Suppress MPNST Growth via JNK. Clin Cancer Res 2019;25:4117-27.

17. Chan DW, Liu VW, Tsao GS, et al. Loss of MKP3 mediated by oxidative stress enhances tumorigenicity and chemoresistance of ovarian cancer cells. Carcinogenesis 2008;29:1742-50.

18. James NE, Beffa L, Oliver MT, et al. Inhibition of DUSP6 sensitizes ovarian cancer cells to chemotherapeutic agents via regulation of ERK signaling response genes. Oncotarget 2019;10:3315-27.

19. James NE, Oliver MT, Ribeiro JR, et al. Human 
Epididymis Secretory Protein 4 (HE4) Compromises Cytotoxic Mononuclear Cells via Inducing Dual Specificity Phosphatase 6. Front Pharmacol 2019;10:216.

20. Wu F, McCuaig RD, Sutton CR, et al. Nuclear-Biased DUSP6 Expression is Associated with Cancer Spreading Including Brain Metastasis in Triple-Negative Breast Cancer. Int J Mol Sci 2019. doi: 10.3390/ijms20123080.

21. Unni AM, Harbourne B, Oh MH, et al. Hyperactivation of ERK by multiple mechanisms is toxic to RTK-RAS mutation-driven lung adenocarcinoma cells. Elife 2018. doi: 10.7554/eLife.33718.

Cite this article as: Sato S, Itamochi H. Dual specificity phosphatase 6 as a new therapeutic target candidate for epithelial ovarian cancer. Ann Transl Med 2019;7(Suppl 8):S373. doi: 10.21037/atm.2019.12.105
22. Albertson DG. Gene amplification in cancer. Trends Genet 2006;22:447-55.

23. Weinstein IB, Joe AK. Mechanisms of disease: Oncogene addiction--a rationale for molecular targeting in cancer therapy. Nat Clin Pract Oncol 2006;3:448-57.

24. Sharma SV, Settleman J. Oncogene addiction: setting the stage for molecularly targeted cancer therapy. Genes Dev 2007;21:3214-31.

25. Kaelin WG Jr. The concept of synthetic lethality in the context of anticancer therapy. Nat Rev Cancer 2005;5:689-98. 